\title{
Quantify Glucose Level in Freshly Diabetic's Blood by Terahertz Time-Domain Spectroscopy
}

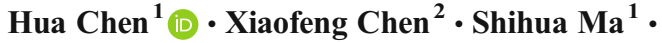 \\ Xiumei $\mathrm{Wu}^{1}$ - Wenxing Yang ${ }^{1}$ - Weifeng Zhang ${ }^{2}$. \\ $\mathrm{Xiao} \mathbf{L i}^{2}$
}

Received: 6 October 2017 / Accepted: 22 December 2017 /

Published online: 15 February 2018

(C) The Author(s) 2018. This article is an open access publication

\begin{abstract}
We demonstrate the capability of terahertz (THz) time-domain spectroscopy (TDS) to quantify glucose level in ex vivo freshly diabetic's blood. By investigating the THz spectra of different human blood, we find out $\mathrm{THz}$ absorption coefficients reflect a high sensitivity to the glucose level in blood. With a quantitative analysis of 70 patients, we demonstrate that the $\mathrm{THz}$ absorption coefficients and the blood glucose levels perform a linear relationship. A comparative experiment between $\mathrm{THz}$ measurement and glucometers is also conducted with another 20 blood samples, and the results confirm that the relative error is as less as $15 \%$. Our ex vivo human blood study indicates that $\mathrm{THz}$ technique has great potential application to diagnose blood glucose level in clinical practice.
\end{abstract}

Keywords THz-TDS · Absorption coefficients · Human blood $\cdot$ Glucose level

\section{Introduction}

Recently THz-TDS has been proved to be a feasible and effective way to detect protein [1-3], DNA [4, 5], biopolymers [6], and biomolecules [7, 8] et al. This technology opens potentially many applications for $\mathrm{THz}$ spectroscopy $[8,9]$ such as biomedicine, pharmaceutical analysis, real-time monitoring of biological processes, and detecting and identification of harmful biological species [10-13]. In the past, THz-TDS has been used to investigate the dielectric properties of the ex vivo blood of human and rat [14-19]. This opens a new way to diagnose blood glucose level in diabetes.

Hua Chen

chenhua@seu.edu.cn

1 School of Physics, Southeast University, Nanjing, Jiangsu 211189, China

2 The First Affiliated Hospital of Nanjing Medical University, Nanjing, Jiangsu 210029, China 
Diabetes is a group of metabolic diseases in which a person has high blood glucose level. This high blood glucose level produces the classical symptoms of polyuria, polydipsia, and polyphagia. In clinical practice, for the majority of healthy individuals, normal blood glucose levels are under $108 \mathrm{mg} / \mathrm{dl}$ when fasting and up to $140 \mathrm{mg} / \mathrm{dl}$ after 2-h post-prandial. For people with diabetes, blood glucose levels are more than $126 \mathrm{mg} / \mathrm{dl}$ when fasting and $200 \mathrm{mg} / \mathrm{dl}$ or more after 2-h post-prandial. In 2015, according to the International Diabetes Federation (IDF), at least 415 million people worldwide suffer from diabetes. Its incidence is increasing rapidly, and it is estimated that by 2030, this number will almost double [20]. In our previous study [15], based on the THz absorption coefficient spectra study on human's bloods, we demonstrated that the $\mathrm{THz}$ absorption coefficients and the blood glucose levels perform a linear relationship. However, only less than 12 samples were studied and the relative error was high. Here, with 70 freshly diabetic's bloods, we further study the effect of blood glucose level on the $\mathrm{THz}$ absorption properties. Our results confirm that the $\mathrm{THz}$ absorption coefficients of blood vary at different glucose level, and they perform as a linear relationship with the relative error as less as $15 \%$. Meanwhile, the capability of THz-TDS to quantify the blood glucose level without any other methods is also confirmed by a comparative experiment between $\mathrm{THz}$ measurement and glucometers with another 20 blood samples. Our investigation demonstrates that $\mathrm{THz}$ wave has the potential application to clinical human blood glucose level diagnostics.

\section{Experimental Setup}

A standard terahertz time-domain spectroscopy apparatus for transmission measures is used. The setup and corresponding experimental data analysis method has been discussed in detail in our previous reports $[21,22]$. In brief, the THz-ray is generated by the $\left(\begin{array}{lll}1 & 0 & 0\end{array}\right)$-oriented InAs wafer, which is excited by optical pulses from a mode-locked Ti-sapphire laser $(\lambda=800 \mathrm{~nm}$, $\tau_{\mathrm{FWHM}}=100 \mathrm{fs}$ ). The repetition rate of the laser is $82 \mathrm{MHz}$. The THz radiation from the InAs is collimated and focused onto the sample layer by means of the off-axis parabolic mirrors. Using another parabolic mirror, the transmitted $\mathrm{THz}$ pulses through the sample are again collimated and focused onto the detector, which is an electric-optic (EO) crystal ZnTe. Probe beam, derived from the same optical source by a half mirror, is employed to gate the detector. We generate and detect the THz signal in nitrogen condition to get rid of the influence of water vapor on the spectra.

An Omni Cell ${ }^{\mathrm{TM}}$ System (Specac Limited, UK), as shown in Fig. 1, is used as a sample chamber for transmission measurement. The liquid sample system is with two polythene (PE) cell windows and a 200- $\mu$ m-thick Teflon chamber. They are sandwiched by two plates and locked by four quick release nuts. After the liquid blood sample being injected into one filling port, we seal the port by luer plugs. During $\mathrm{THz}$ measurement, the room temperature is kept at $23^{\circ} \mathrm{C}$ with a stable humidity $50 \%$.

At first, we measured pure water (tap water) with this liquid sample system for repeatability test, and the test number was five. After one test done, we changed water for the next test. Figure 2 shows the absorption coefficient spectra of water. The data was acquired between 0.2 and $0.9 \mathrm{THz}$, and the high water absorption made the data unreliable in the higher $\mathrm{THz}$ frequency. The results based on five tests indicate 
Fig. 1 Omni Cell ${ }^{\mathrm{TM}}$ System

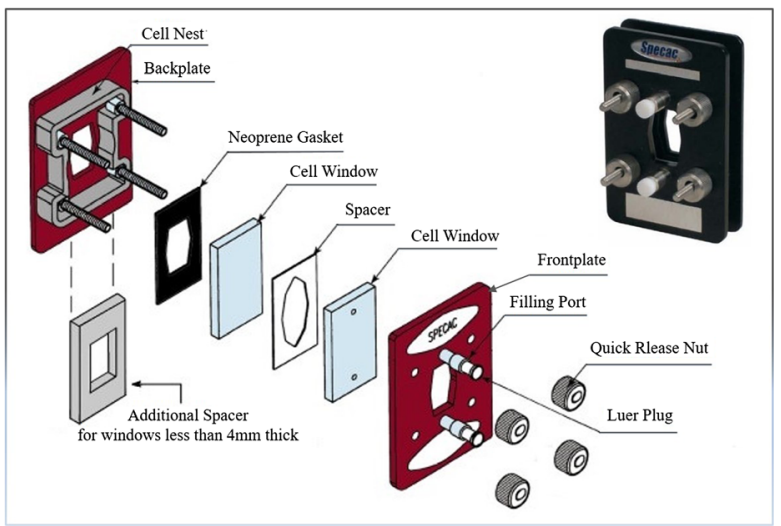

that our measurement has a high repeatability, and the ratio of the standard deviation to the average value was as low as $3 \%$.

\section{Sample Preparation and Blood Spectra Acquisition}

In 3 years, we have measured 70 different human's blood samples (mean age 61 years; range 51-82 years), and these samples were provided by Nanjing Medical University, Nanjing, China. The freshly extracted blood samples from diabetes were first injected into heparin vacutainer and we added heparin as anticoagulant (15 US Pharmacopeia units of heparin per milliliter of blood) [23]. And then, we brought the samples into THz measurement within half an hour. During the carrying, we kept the samples in cold storage condition $\left(\approx 4{ }^{\circ} \mathrm{C}\right)$. Before the experiment, we raised the temperature of the sample to the room temperature by soaking the vacutainer in $25^{\circ} \mathrm{C}$ warm water for $5 \mathrm{~min}$.

For each measurement, a reference waveform was taken with the empty chamber. All the acquired sample and reference waveforms were converted to their Fourier transforms $E_{s}(\omega)$

Fig. 2 Five-times measured absorption coefficients of tap water. The error bars represent the standard deviation of the mean and the test number is 5

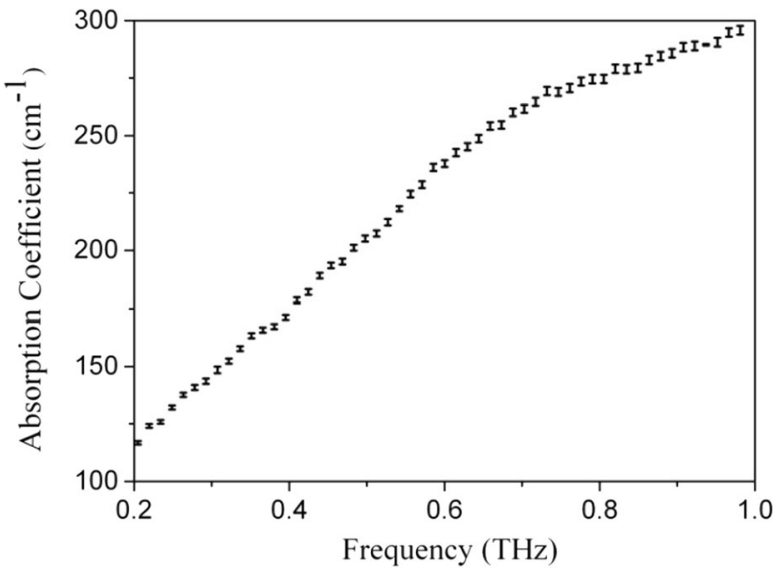


and $E_{\text {ref }}(\omega)$, respectively. Then the THz electric field transmitted through the empty chamber $E_{\text {ref }}(\omega)$ is:

$$
E_{r e f}(\omega)=E_{s}(\omega) \cdot T(\omega)_{(\mathrm{PE}-\mathrm{air})} \cdot e^{(i \omega \cdot d / c)} \cdot T(\omega)_{(\mathrm{PE}-\mathrm{air})},
$$

where $E_{s}(\omega)$ is the electric field of the THz source, $\omega$ is the THz angular frequency, $d$ is the thickness of the fluidic chamber, and $c$ is light speed. $T(\omega)$ is the transmission coefficient; And the electric field transmitted through the chamber with blood $E_{\text {sam }}(\omega)$ is:

$$
E_{\text {sam }}(\omega)=E_{S}(\omega) \cdot T(\omega)_{(\mathrm{PE}-\mathrm{blood})} \cdot e^{(i \omega \cdot d \cdot n(\omega) / c)} \cdot e^{(-\omega \cdot d \cdot \kappa(\omega) / c)} \cdot T(\omega)_{(\mathrm{PE}-\mathrm{blood})},
$$

where $n(\omega)$ and $\kappa(\omega)$ are the real and imaginary parts of the refractive index of blood. $T(\omega)_{1-2}$, which is the transmission coefficient from media 1 to media 2 , has the form of $\frac{2 \overline{n_{1}}}{\overline{n_{1}}+\overline{n_{2}}}$, where $\bar{n}=n+i \kappa$ is the complex refractive index. The THz absorption in air is neglected. For the refractive indices of PE and air are 1.5 [24] and 1, respectively, and the absorption of PE is negligible [24]. Therefore, the complex refractive index of blood can approximately be described as [25-27]:

$$
\begin{aligned}
& \operatorname{kblood}(\omega) \approx\left[\frac{\ln \left(\left|\frac{E_{\text {sam }}(\omega)}{E_{r e f}(\omega)}\right|^{2}\right)}{d}\right] \times \frac{c}{2 \omega}, \\
& n_{\text {blood }}(\omega) \approx\left(\Delta \varnothing_{\text {sam-ref }}\right) \times c / \omega d+1,
\end{aligned}
$$

where $\left(\Delta \emptyset_{\text {sam-ref }}\right)$ is the phase difference between the sample and the reference. With the knowledge of sample thickness $d$, the absorption coefficient $\alpha$ can be calculated based on the Beer-Lambert law [27]. We substitute the approximate $n_{\text {blood }}$ and $\kappa_{\text {blood }}$ calculated by Eq. (3) and Eq. (4) back to Eq. (2). The transmission coefficients through interfaces can thus be eliminated to keep the blood induced absorption exponential term and to calculate new $n_{\text {blood }}$ and $\kappa_{\text {blood }}$. By repeating this process several times, the $n_{\text {blood }}$ and $\kappa_{\text {blood }}$ will converge to fixed values. As the converged $n_{\text {blood }}$ and $\kappa_{\text {blood }}$ values had less than 0.001 variations, the iteration process stopped. The absorption coefficient $\alpha$ is calculated as:

$$
\alpha=2 \cdot \omega \cdot \frac{\kappa(\omega)}{c}=-\frac{\ln \left(\left|\frac{E_{\text {sam }}(\omega)}{E_{\text {ref }}(\omega)}\right|^{2}\right)}{d},
$$

Figure $3 \mathrm{a}$ shows one time domain waveforms of the $\mathrm{THz}$ pulse measured after the empty chamber and two corresponding traces measured after the chamber filled with blood. The measured $\mathrm{THz}$ waveforms show a main pulse that comes from the direct transmission through the sample, but there also is a smaller pulse following the main pulse caused by the internal Fabry-Pérot (FP) reflection in the PE windows [28]. In our experiments, the smaller reflected pulses are well separated from the main pulses. These echoes are truncated from the measured terahertz waveforms by restricting the waveforms within a finite time window. Therefore, the effect of reflection in the PE windows on the absorption coefficient could be neglected. The glucose level of two blood samples is 105.8 and $136.9 \mathrm{mg} / \mathrm{dl}$, respectively, which are measured by glucometers. The corresponding frequency-domain waveforms obtained by the fast 

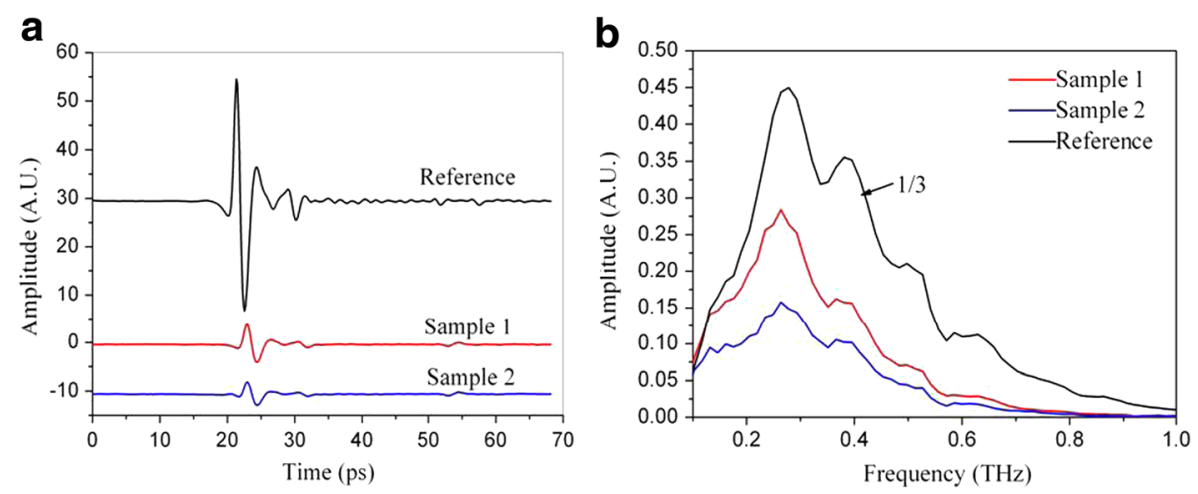

Fig. 3 a The time-domain waveforms of THz wave transmitting through the empty chamber and two corresponding traces measured after the chamber filled with blood. The offset vertical is used for clarity. $\mathbf{b}$ The corresponding frequency-domain spectra of the traces shown in a. For clarity, the amplitude of the reference is divided by 3

Fourier transform are shown in Fig. 3b. Figure 4 shows the calculated absorption coefficient spectra of two blood samples. As previously mentioned, the blood chamber is $200 \mu \mathrm{m}$ thick. Based on a quick calculation, a round trip distance of the $\mathrm{THz}$ wave in the sample cell was $400 \mu \mathrm{m}$. The corresponding FP effect [28] occurrence frequency was theoretically above $0.75 \mathrm{THz}$. From the spectra, we observe that the occurrence of FP effect starts from $0.60 \mathrm{THz}$. Therefore, the effective bandwidth of the measurement system is from 0.20 to $0.50 \mathrm{THz}$. It is clear that the blood sample with higher glucose level absorbs more THz wave and the absorption coefficients increased with the frequency. The distinguishable absorption contrast is the key to realize using $\mathrm{THz}$ wave to quantify the glucose level in human blood. As we known, the free water molecules have larger absorption than the other solute molecules in $\mathrm{THz}$ frequency range and the absorption coefficients decrease with the concentration of the solute $[29,30]$. This is caused by the fact that some of water molecules, which strongly absorb $\mathrm{THz}$ radiation, are replaced by solute molecules less absorbing in this frequency range. However, our results in Fig. 4 showed that diabetic's blood is a very complicated fluid which has the THz spectral characteristics different from the predicted ones of the simple aqueous solutions.

\section{Correlation Analysis}

Other factors such as proteins (including albumin and globulin), ions (including $\mathrm{Na}^{+}, \mathrm{Ca}^{2+}$, and $\mathrm{K}^{+}$), lipids et al. would also modify the $\mathrm{THz}$ absorption coefficients in aqueous solutions [4], so we use a Pearson correlation analysis method to investigate the correlation between $\mathrm{THz}$ absorption coefficient and the factors (glucose, $\mathrm{Na}+, \mathrm{Ca}^{2+}, \mathrm{K}^{+}, \mathrm{TG}, \mathrm{RBC}, \mathrm{HB}, \mathrm{WBC}$ ) in the blood. The sample correlation coefficient $\mathrm{R}$ of the data pairs $\left(x_{i}, y_{i}\right)$ is defined as [31]:

$$
R=\frac{\sum_{i=1}^{n}\left(x_{i}-\bar{x}\right)(y-\bar{y})}{(n-1) S_{x} S_{y}}=\frac{\sum_{i=1}^{n}\left(x_{i}-\bar{x}\right)(y-\bar{y})}{\sqrt{\sum_{i=1}^{n}\left(x_{i}-\bar{x}\right)^{2} \sum_{i=1}^{n}(y-\bar{y})^{2}}}, i=1,2, \ldots \ldots, \mathrm{n}
$$


Fig. 4 The calculated absorption coefficient spectra of two blood samples

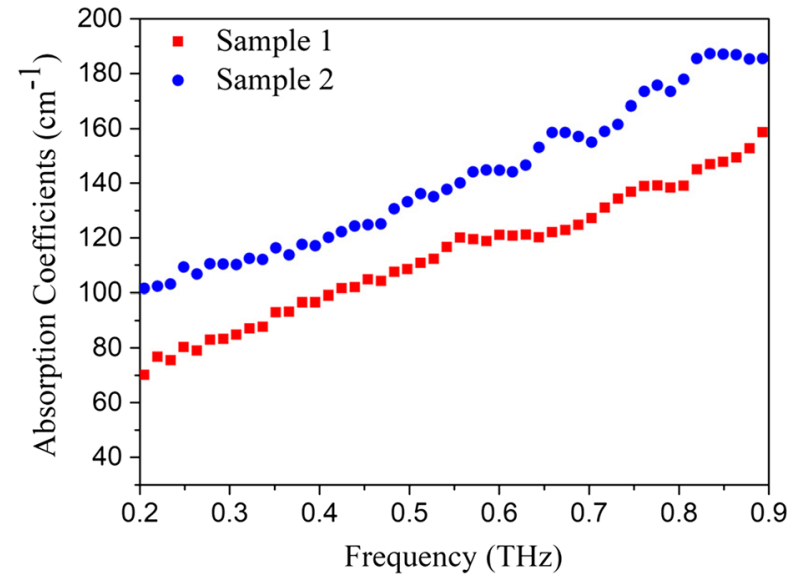

In our analysis, $x$ is the absorption coefficient, and $y$ is the examined variable. If $R>0$, it means that the sample pairs are positively correlated, and if $R<0$, it means they are negatively correlated. Meanwhile, the absolute value of $R(|R|)$ is a measure of the strength of the linear relationship between the $x$ and $y$ value of a data pair. A value of $|R|$ equal to 1 means that there is a perfect linear relation - that is, a straight line can pass through all the data point. $|R|>0.5$ represents a moderate to strong relationship, $0.3<|R|<0.5$ represents a weak to moderate relationship, and $|R|<0.3$ represents a weak relationship [31]. This bivariate correlation analysis between the examined items and the THz absorption coefficient was done by a Statistical Product and Service Solutions (SPSS) program [32].

Considering the system bandwidth limitation, a frequency range of 0.2 to $0.5 \mathrm{THz}$ is accurate for subsequent analysis. Based on the blood health examination reports and the corresponding $\mathrm{THz}$ absorption coefficients of the same whole blood samples, we subject the data to Eq.6. The statistical number is 20. In Table 1, we show the correlation coefficients between $\mathrm{THz}$ absorption coefficient (at $0.2,0.35$, and $0.5 \mathrm{THz}$ ) and glucose level and other factors in blood. It is clear that the THz absorption coefficient is highly correlated with the glucose level. But there are also some outlier factors affecting the THz wave absorption from the correlation analysis result. So we use the stepwise regression analysis method $[33,34]$ to find out the outlier factors in each frequency. In regression analysis, we got the unary and

Table 1 Correlation between $\mathrm{THz}$ absorption coefficient and blood health examination items

\begin{tabular}{lcrr}
\hline Examination items & \multicolumn{2}{l}{ Frequency } & \\
\cline { 2 - 4 } & $0.20 \mathrm{THz}$ & $0.35 \mathrm{THz}$ & $0.50 \mathrm{THz}$ \\
\hline Glucose $(\mathrm{mg} / \mathrm{dl})$ & 0.78 & 0.91 & 0.94 \\
$\mathrm{Na}^{+}(\mathrm{mmol} / \mathrm{l})$ & -0.52 & -0.54 & -0.47 \\
$\mathrm{Ca}^{2+}(\mathrm{mmol} / \mathrm{l})$ & -0.25 & 0.03 & 0.13 \\
$\mathrm{~K}^{+}(\mathrm{mmol} / \mathrm{l})$ & 0.28 & 0.22 & 0.25 \\
$\mathrm{TG}(\mathrm{mg} / \mathrm{dl})$ & 0.06 & 0.25 & 0.17 \\
$\mathrm{RBC}(\mathrm{M} / \mu \mathrm{l})$ & -0.57 & -0.33 & -0.09 \\
$\mathrm{HB}(\mathrm{g} / \mathrm{dl})$ & -0.52 & 0.31 & -0.15 \\
$\mathrm{WBC}(\mathrm{K} / \mu \mathrm{l})$ & -0.25 & 0.03 & 0.18 \\
\hline
\end{tabular}

$T G$ triglyceride, $R B C$ red blood cell, $H B$ hemoglobin, $W B C$ white blood cell 
multiple linear regression equation. By comparing the results of linear regression, Table 2 shows the main factor and the outlier factors which affecting $\mathrm{THz}$ wave absorption in each frequency, and glucose level is the most related and dominant factor. The $\mathrm{HB}$ and $\mathrm{Na}^{+}$become outlier factors when the frequency decreased. At $0.5 \mathrm{THz}$, we also find that no other blood factors would directly affect $\mathrm{THz}$ wave absorption. Therefore, the quantification of glucose level in diabetic's blood in Section 5 will be investigated at $0.5 \mathrm{THz}$.

\section{Quantitative Analysis}

Based on 70 diabetes patients' blood, we investigated the relationship between the $\mathrm{THz}$ absorption coefficients and the glucose levels. Figure 5 is the correlating figure of $\mathrm{THz}$ wave absorption coefficients and the corresponding glucose level of patients' blood at $0.5 \mathrm{THz}$, which is in the middle of our acquired frequency region $(0.1$ and $0.9 \mathrm{THz})$. By fitting the data, we find out the $\mathrm{THz}$ absorption coefficients and the blood glucose levels perform a linear relationship as Glu $=0.55+0.99 \alpha$, while Glu is glucose level in blood. This linear correlation indicates that quantitative blood glucose level analysis is feasible by using THz-TDS.

\section{Performance Study and Discussion}

With this excellent linear relationship, we try to test the capability of THz-TDS to quantify blood glucose levels directly. In this study, we choose another 20 blood samples and divided every blood sample into two parts. One part is for THz-TDS measurement, and the other part for glucometer (Roche Accu-chek Perfoma glucometer, standard error $\pm 15 \%$ ) measurement. We perform these two measurements separately, that is to say, before THz-TDS measurement, we did not know the blood glucose levels of these 20 samples. After we measured this part of 20 blood samples by THz-TDS and calculated their corresponding absorption coefficients at $0.5 \mathrm{THz}$, we calculated the glucose levels according to the linear relationship Glu $=0.55+$ $0.99 \alpha$. And then, we measured the other part of the 20 blood samples by glucometers. Finally, we compare these two set of results in Table 3. It is clear that the results from these two measurements match well with each other. From the comparison results of these two measurements, we find the relative error is as less as $15 \%$. Therefore, we demonstrate that THzTDS has the capability to quantify glucose level in human blood without any other methods.

The performance study is an important first step for application to human clinical examination and would encourage broad study on $\mathrm{THz}$ non-invasive diagnose diabetes. In the future study, we will develop a new THz imaging system to scan the patient's fingers or ears, which are all good sources of capillary. With the significant linear correlation between $\mathrm{THz}$ absorption coefficients and the glucose levels, we would directly measure the blood glucose levels. In clinical practice, the blood glucose level measurements are mostly performed by glucometers.

Table 2 Main factor and outlier factors affecting the $\mathrm{THz}$ wave absorption

\begin{tabular}{lll}
\hline Frequency & Main factor & Outlier factors \\
\hline $0.20 \mathrm{THz}$ & Glucose & $\mathrm{HB}$ \\
$0.35 \mathrm{THz}$ & Glucose & $\mathrm{Na}^{+}$ \\
$0.50 \mathrm{THz}$ & Glucose & None \\
\hline
\end{tabular}


Fig. 5 The correlation of $\mathrm{THz}$ wave absorption coefficients and the corresponding blood glucose level of human blood

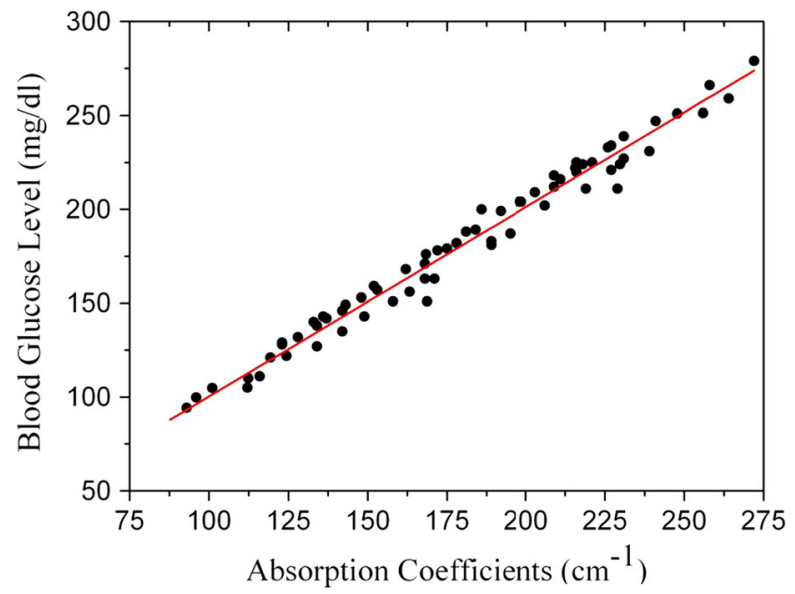

This method requires that a patient makes a finger puncture for every measurement. About seven punctures per day and at least three times per week should be done for proper glucose monitoring. It means one patient will suffer about 1000 punctures per year. The constant skin damage and pain cannot be avoided. Besides, each measurement by glucometer requires a distinct test strip. A large expenditure for 1000 test strips is essential. It is also necessary to consider that each puncture has a risk of blood infection. Therefore, a non-invasive $\mathrm{THz}$ examination could not only alleviate the suffering of patients and reduce the amount of possible risky manipulations, but also economize the use of hospital and human resources.

Table 3 Glucose levels measured by THz-TDS and glucometers

\begin{tabular}{|c|c|c|}
\hline \multicolumn{2}{|l|}{ Method } & \multirow[t]{2}{*}{ Relative error $(\%)$} \\
\hline THz-TDS (mg/dl) & Glucometers (mg/dl) & \\
\hline 89.9 & 81.7 & 9.1 \\
\hline 95.5 & 87.2 & 8.7 \\
\hline 103.0 & 91.7 & 11.0 \\
\hline 106.8 & 97.4 & 8.8 \\
\hline 108.7 & 102.3 & 5.9 \\
\hline 112.4 & 105.0 & 6.6 \\
\hline 118.0 & 108.6 & 8.0 \\
\hline 121.8 & 114.3 & 6.2 \\
\hline 129.3 & 119.7 & 7.4 \\
\hline 131.2 & 126.6 & 3.6 \\
\hline 140.6 & 152.9 & 8.7 \\
\hline 142.4 & 158.8 & 11.5 \\
\hline 149.9 & 160.2 & 6.9 \\
\hline 151.8 & 172.1 & 13.4 \\
\hline 159.3 & 169.5 & 6.4 \\
\hline 161.2 & 181.4 & 12.5 \\
\hline 168.7 & 188.8 & 11.9 \\
\hline 170.6 & 194.7 & 14.1 \\
\hline 176.2 & 191.2 & 8.5 \\
\hline 178.0 & 189.1 & 6.2 \\
\hline
\end{tabular}




\section{Conclusion}

In conclusion, based on the $\mathrm{THz}$ absorption coefficient spectra study of 70 human bloods, we not only demonstrate that a different blood glucose level has individual $\mathrm{THz}$ absorption ability, but also demonstrate that the THz absorption coefficients and the blood glucose levels perform an excellent linear relationship. The capability of THz-TDS to quantify the blood glucose level without any other methods is also confirmed. Our research provides a fast, accurate, and continuous method to measure glucose lever in diabetic's blood, which has great potential application in clinical practice.

Funding information This work was supported by Joint Research Project by Southeast University and Nanjing Medical University Nos. 3207027381 and China Postdoctoral Science Foundation Nos. 2016 M601692.

Open Access This article is distributed under the terms of the Creative Commons Attribution 4.0 International License (http://creativecommons.org/licenses/by/4.0/), which permits unrestricted use, distribution, and reproduction in any medium, provided you give appropriate credit to the original author(s) and the source, provide a link to the Creative Commons license, and indicate if changes were made.

\section{References}

1. Seung Jae Oh, Yong-Min Huh, Jin-Suck Suh, Jihye Choi, Seungjoo Haam, Joo-Hiuk Son, Cancer diagnosis by terahertz molecular imaging technique, Journal of Infrared, Millimeter, and Terahertz Waves, 33(1), 74 81, (2012).

2. Tyler Bowman, Yuhao Wu, John Gauch, Lucas K. Campbell, Magda El-Shenawee, Terahertz imaging of three-dimensional dehydrated breast cancer tumors, Journal of Infrared, Millimeter, and Terahertz Waves, 38(6), 766-786, (2017).

3. Yuichi Ogawa, Shin'ichiro Hayashi, Masato Oikawa, Chiko Otani, and Kodo Kawase, Interference terahertz label-free imaging for protein detection on a membrane, Opt. Express, 16(26), 22083-22089 (2008).

4. A. G. Markelz, A. Roitberg, and E. J. Heilweil, Pulsed terahertz spectroscopy of DNA, bovine serum albumin and collagen between 0.1 and 2.0 THz, Chemical Physics Letters, 320(1-2), $42-48$ (2000).

5. B. M. Fischer, M. Walther, and P. U. Jepsen, Far-infrared vibrational modes of DNA components studied by terahertz time-domain spectroscopy, Physics in Medicine and Biology, 47(21), 3807-3814 (2002).

6. N. Nagai, and Y. Katsurazawa, Analysis of the inter-molecular interactions between amino acids and acetone by THz spectroscopy, Biopolymers, 85(3), 207-213 (2007).

7. R. M Woodward, V. P Wallace, D. D. Arnone, E. H. Linfield, and M. Pepper, Terahertz pulsed imaging of skin cancer in the time and frequency domain, Journal of Biological Physics, 29(2-3) 257-259 (2003).

8. T. Loffler, T. Bauer, K. Siebert, H. Roskos, A. Fitzgerald, and S. Czasch, Terahertz dark-field imaging of biomedical tissue, Opts. Express, 9(12), 616-621 (2001)

9. B. Ferguson, S. Wang, D. Gray, D. Abbott, and X. C. Zhang, Identification of biological tissue using chirped probe THz imaging, Microelectronics Journal, 33(12), 1043-1051 (2002).

10. R. M. Woodward, B. E. Cole, V. P. Wallace, R. J. Pye, D. D. Arnone, E. H. Linfield, and M. Pepper, Terahertz pulse imaging in reflection geometry of human skin cancer and skin tissue, Phys. Med. Biol., 47(21), 3853-3863 (2002).

11. D. Crawley, M. Pepper, V. P. Wallace, B. Cole, D. Arnone, and C. Longbottom, Three-dimensional terahertz pulse imaging of dental tissue, J. Biomed. Opt., 8(2), 303-307 (2003).

12. S. Huang, P. C. Ashworth, K. W. C. Kan, Y. Chen, V. P. Wallace, Y. Zhang, and Emma PickwellMacPherson, Improved sample characterization in terahertz reflection imaging and spectroscopy, Opts. Express, 17(5), 3848-3854 (2009)

13. H. Chen, Chui-Min Chiu, Wei-Ling Lai, Yuan-Fu Tsai, Tzu-Fang Tseng, Jen-Tang Lu, Wen-Jeng Lee, HsinYi Huang, Chung-Wu Lin, and Chi-Kuang Sun, Performance of THz fiber-scanning near-field microscopy to diagnose breast tumors, Optics Express, 19(20), 19523 (2011) 
14. D. K. Kim, J.-H. Won, V. V. Meriakri, and E. E. Chigrai, Device for the non-invasive measurement of blood glucose concentration by millimeter waves and method thereof, US patent 7371217 B2, Samsung Electronics Co., Ltd.

15. C.-K. Sun, Y.-F. Tsai, and H. Chen, Method and device for detecting a blood glucose level using an electromagnetic wave, US patent 20130289370 A1, National Taiwan University.

16. C. Reid, G. Reese, A.P. Gibson, and V. P. Wallace, Terahertz time domain spectroscopy of human blood, IEEE J. Biomed. Health Inform., 17(4), 774-778 (2013)

17. K. Jeong, Y.-M. Huh, S.-H. Kim, Y. Park, J.-H. Son, S.J. Oh, and J.-S. Suh, Characterization of blood using terahertz waves, J. Biomed. Opt., 18(10), 107008 (2013)

18. O. P. Cherkasova, M. M. Nazarov, I. N. Smirnova, A. A. Angeluts, and A. P. Shkurinov, Application of time-domain THz spectroscopy for studying blood plasma of rats with experimental diabetes, Phys. Wave Phenom. 22(3), 185-188 (2014).

19. Tzu-Fang Tseng, Borwen You, Hao-Cheng Gao, Tzung-Dau Wang, and Chi-Kuang Sun, Pilot clinical study to investigate the human whole blood spectrum characteristics in the sub-THz region, Opt. Express, 23(7), 9440-9451 (2015)

20. S. Wild, S. Wild, G. Roglic, A. Green, R. Sicree, and H. King, Global prevalence of diabetes: estimates for 2000 and projections for 2030, Diabetes Care, 27(5), 1047-1053 (2004).

21. H. Chen, L. Wang, Y. G. Qu, T. Y. Kuang, L. B. Li, and W. X. Peng, Investigation of guanidine hydrochloride induced chlorophyll protein 43 and 47 denaturation in the terahertz frequency range, J. App. Phys., 102(7), 074701:1-3 (2007).

22. H. Chen, G. Y. Chen, S. Q. Li and L. Wang, Reversible conformational changes of PsbO protein detected by terahertz time-domain spectroscopy, Chinese Physics Letter 26(8), 054209 (2009).

23. J. J. Cerón, S. Martínez-Subiela, C. Hennemann, and F. Tecles, The effects of different anticoagulants on routine canine plasma biochemistry, Vet. J., 167(3), 294-301 (2004).

24. B. Ung, A. Dupuis, K. Stoeffler, C. Dubois, and M. Skorobogatiy, High-refractive-index composite materials for terahertz waveguides: trade-off between index contrast and absorption loss, J. Opt. Soc. Am. B, 28(4), 917-921 (2011).

25. W. Withayachumnankul, and M. Naftaly, Fundamentals of measurement in terahertz time-domain spectroscopy, Journal of Infrared Millimeter and Terahertz Waves, 35(8):610-637 (2014).

26. S. A. Maier, Plasmonics: fundamentals and applications, Springer, Berlin, pp 49-74, (2014).

27. G. M. Png, J. W. Choi, B. W.-H. Ng, S. P. Mickan, D. Abbott, and X.-C. Zhang, The impact of hydration changes in fresh bio-tissue on THz spectroscopic measurements, Phys. Med. Biol. 53(13), 3501-3517 (2008).

28. H. A. Haus, Waves and fields in optoelectronics, Prentice Hall, Upper Saddle River (1984).

29. Takashi Arikawa, Masaya Nagai, and Koichiro Tanaka, Characterizing hydration state in solution using terahertz time-domain attenuated total reflection spectroscopy, Chem. Phys. Lett. 457(20), 12-17 (2008).

30. Z. F. Jiang, Y.X. Wang, X. H. Li, C. Z. Lu, W. Liu, P. Sun, and L. Miao, Terahertz time-domain spectroscopy of D-glucose in the solution states, Proc. SPIE 8910, international symposium on photoelectronic detection and imaging 2013: imaging spectrometer technologies and applications, 89101T (2013).

31. S. M. Ross, Introduction to probability and statistics for engineers and scientists, 3 ed., Wiley, Hoboken (2004).

32. SPSS software introduction, (http://www-01.ibm.com/software/analytics/spss/).

33. J. Cohen and P. Cohen, Applied multiple regression/correlation analysis for the behavioral sciences, L. Erlbaum Associates, Mahwah (2003).

34. B. G. Tabachnick and L. S. Fidell, Using multivariate statistics: Pearson new international edition, Pearson Schweiz Ag, Zug (2012). 